\title{
Prevalence of latent tuberculosis infection and risk of infection in patients with chronic kidney disease undergoing hemodialysis in a referral center in Brazil*
}

\author{
Prevalência de infecção latente por Mycobacterium tuberculosis e risco de \\ infecção em pacientes com insuficiência renal crônica em hemodiálise em \\ um centro de referência no Brasil
}

\author{
Jane Corrêa Fonseca, Waleska Teixeira Caiaffa, Mery Natali Silva Abreu, \\ Katia de Paula Farah, Wânia da Silva Carvalho, Silvana Spindola de Miranda
}

\begin{abstract}
Objective: To determine the prevalence of latent tuberculosis infection (LTBI) and the risk of infection in patients with chronic kidney disease treated at a hemodialysis center. Methods: We included 307 patients with chronic kidney disease undergoing hemodialysis at the Mineiro Institute of Nephrology, located in the city of Belo Horizonte, Brazil. All of the patients were submitted to tuberculin skin tests (TSTs). We investigated the booster effect and TST conversion. If the initial TST (TST1) was negative, a second TST (TST2) was performed 1-3 weeks later in order to investigate the booster effect. If TST2 was also negative, a third TST (TST3) was performed one year after TST2 in order to determine whether there was TST conversion. Results: When we adopted a cut-off induration of $5 \mathrm{~mm}$, the prevalence of LTB1 was $22.2 \%$ on TST1, increasing by $11.2 \%$ on TST2. When we adopted a cut-off induration of $10 \mathrm{~mm}$, the prevalence of LTB1 was $28.5 \%$ on TST1, increasing by $9.4 \%$ on TST2. The prevalence of LTB1 increased significantly from TST1 to TST2 (booster effect), as well as from TST2 to TST3 ( $p<0.01$ for both). In our sample, the mean annual risk of infection was 1.19\%. Conclusions: In the population studied, the prevalence of LTBl was high, and the mean annual risk of infection was similar to that reported for the general population of Brazil, which suggests recent infection.
\end{abstract}

Keywords: Tuberculosis; Renal insufficiency, chronic; Tuberculin test.

\section{Resumo}

Objetivo: Determinar a prevalência da infecção latente por Mycobacterium tuberculosis (ILMT) e o risco de infecção em pacientes com insuficiência renal crônica em um centro de hemodiálise. Métodos: Foram incluídos no estudo 307 pacientes com insuficiência renal crônica em tratamento hemodialítico no lnstituto Mineiro de Nefrologia, na cidade de Belo Horizonte (MG). Todos os pacientes foram submetidos a testes tuberculínicos (TTs). 0 efeito booster e a viragem tuberculínica foram avaliados. Se o primeiro TT (TT1) era negativo, um segundo (TT2) era realizado 1-3 semanas após o TT1 para investigar o efeito booster. Se o TT2 também era negativo, um terceiro (TT3) era realizado um ano após o TT2 para identificar a viragem tuberculínica. Resultados: A prevalência da ILMT, quando considerado o ponto de corte de $5 \mathrm{~mm}$ de enduração, foi de 22,2\% no TT1, com incremento de 11,2\% no TT2. A prevalência da ILMT, quando considerado o ponto de corte de enduração de $10 \mathrm{~mm}$, foi de 28,5\% no TT1, com incremento de 9,4\% no TT2. Um aumento significativo da prevalência da ILMT foi observado entre TT1 e TT2 (efeito booster) e entre TT2 e TT3 ( $p<0,01$ para ambos). Na nossa amostra, o risco médio anual de infecção foi de 1,19\%. Conclusões: Na população estudada, a prevalência da 1LMT foi alta, e o risco de infecção foi semelhante ao da população geral no Brasil, o que sugere infecção recente.

Descritores: Tuberculose; Insuficiência renal crônica; Teste tuberculínico.

\footnotetext{
* Study carried out at the Mineiro Institute of Nephrology and at the Federal University of Minas Gerais, Belo Horizonte, Brazil. Correspondence to: Silvana Spindola de Miranda. Avenida Alfredo Balena, 190, Faculdade de Medicina/Departamento de Clínica Médica, $2^{\circ}$ andar, Santa Efigênia, CEP 30130-100, Belo Horizonte, MG, Brasil.

Tel. 5531 3248-9599. E-mail: spindola@medicina.ufmg.br

Financial support: This study received financial support from the Federal University of Minas Gerais School of Medicine Graduate Program, the Secretaria Municipal da Saúde de Belo Horizonte (SMSA, Belo Horizonte Municipal Department of Health), the Mineiro Institute of Nephrology, and the Fundação de Amparo à Pesquisa do Estado de Minas Gerais (FAPEMIG, Foundation for the Support of Research in the state of Minas Gerais).

Submitted: 14 September 2012. Accepted, after review: 22 January 2013.
} 


\section{Introduction}

Tuberculosis remains a serious public health problem worldwide. The World Health Organization estimates that there were 50 million people infected with Mycobacterium tuberculosis, 92,000 new cases (incidence, 48/100,000 population), and 49,000 new smear-positive cases (incidence, 26/100,000 population) in Brazil in 2007. In addition, the number of deaths from tuberculosis has been estimated at 8,400 , i.e., 4.4 deaths per 100,000 population. ${ }^{(1)}$

In 2008, the incidence of tuberculosis was 50/100,000 population in Brazil, 23/100,000 population in the state of Minas Gerais, and $31 / 100,000$ population in the city of Belo Horizonte. In southeastern Brazil, the prevalence of tuberculosis is high, which is probably due to worsening of living conditions in urban areas, incorrect diagnosis and treatment, and inappropriate use of available resources..$^{(2,3)}$

Patients with chronic kidney disease (CKD) are immunosuppressed, given that uremia reduces the expression of B7-2 costimulatory molecule on antigen-presenting cells; this alters the function of polymorphonuclear cells and interferes with phagocytic efficiency, migration efficiency, and chemotactic efficiency, reducing the ability of cells to kill intracellular microorganisms. (4) Nevertheless, the tuberculin skin test (TST) remains the standard method for diagnosing latent tuberculosis infection (LTBI) in patients with CKD undergoing hemodialysis and in kidney transplant candidates, although anergy rates vary widely. ${ }^{(5-9)}$

In developed countries, the most common causes of entry into renal replacement therapy programs are diabetes mellitus (DM) and systemic arterial hypertension (SAH), which account for approximately $70 \%$ of all admissions to dialysis centers. In developing countries, there is a higher prevalence of nephropathy related to infectious diseases, although the available data are inaccurate. ${ }^{(10)}$

In patients with CKD undergoing hemodialysis, the risk of developing tuberculosis is 6.9-52.5 times as high as it is in the general population, which is why all patients presenting with positive TST results should receive treatment for LTBI. ${ }^{(11)}$

The objective of the present study was to determine the prevalence of LTBI and the risk of infection in patients with CKD treated at a hemodialysis center.

\section{Methods}

This was a prospective longitudinal study conducted at the Mineiro Institute of Nephrology, located in the city of Belo Horizonte, Brazil. All patients over 18 years of age and undergoing hemodialysis between December of 2008 and December of 2009 were invited to participate in the study.

The study participants completed a standardized, previously validated questionnaire on the following: date of hemodialysis initiation; sociodemographic data (including gender, age, race, and marital status); smoking; alcohol consumption; body mass index; presence of DM; HIV, HBV, and HCV serology; and use of immunosuppressants. In addition, the participants were evaluated in terms of the following: presence of a BCG vaccination scar; history of tuberculosis; and history of contact with a tuberculosis patient.

The etiology of kidney disease and the use of medications (antihypertensives, recombinant human erythropoietin, iron, insulin, diuretics, antidepressants, immunosuppressants, vasodilators, bronchodilators, B vitamins, vitamin C, folic acid, and vitamin D analogs) were reported and evaluated in terms of their association with TST results.

Patients who failed to return for the reading of the TST results, those who had previously undergone a TST, those who had previously been treated for LTBI, and those who presented with a history of tuberculosis were excluded from the present study.

All of the study participants underwent chest X-ray.

The present study was approved by the Research Ethics Committee of the Federal University of Minas Gerais (ETIC Ruling no. 25/08), located in the city of Belo Horizonte, Brazil.

The TST was performed by the Mantoux method, which consists of intradermal administration of $0.1 \mathrm{~mL}$ (2 tuberculin units) of PPD RT23 (State Serum Institute, Copenhagen, Denmark) on the volar aspect of the forearm. The results of the test were read within 72-96 h after PPD RT23 administration. The maximum transverse diameter of the area of palpable induration was measured with a millimeter ruler, and the results were expressed in $\mathrm{mm}$. If the initial TST (TST1) was negative, a second TST (TST2) was performed 1-3 weeks later in order to investigate the booster effect. If TST2 was also negative, a 
third TST (TST3) was performed one year after TST2 in order to determine whether there was TST conversion. ${ }^{(12)}$

We used two cut-off points for TST induration. In general, for patients with CKD, a positive TST result was defined as an induration $\geq 10 \mathrm{~mm}$. For kidney transplant candidates, HIV-positive patients, immunosuppressed patients, patients using prednisone or equivalent at a dose $\geq 15$ $\mathrm{mg} /$ day for more than one month, and candidates for the use of TNF- $\alpha$ inhibitors, a positive TST result was defined as an induration $\geq 5 \mathrm{~mm}$. The latter cut-off point was chosen on the basis of recommendations made by the American Thoracic Society and other authors, ${ }^{(13,14)}$ given that patients receiving immunosuppressants after transplantation have a high risk of developing active tuberculosis.

The booster effect was defined as a TST2 induration $\geq 10 \mathrm{~mm}$, the TST2 induration having increased by more than $6 \mathrm{~mm}$ in comparison with the TST1 induration. ${ }^{(3)}$

We defined TST conversion as a TST3 induration $\geq 10 \mathrm{~mm}$ in comparison with the TST2 induration. ${ }^{(3)}$

For all of the individuals with LTB1, we recommended treatment with isoniazid at a dose of $10 \mathrm{mg} / \mathrm{kg}$ per day (maximum dose, $300 \mathrm{mg} /$ day) after hemodialysis.

In order to calculate the mean annual risk of infection (MARI), we used the formula proposed by Ruffino-Netto. ${ }^{(15)}$

Descriptive statistics were calculated for all study variables. For categorical variables (such as race and gender), we employed frequency distribution tables. For continuous variables (such as age and weight), we adopted measures of central tendency (mean and median) and variability (minimum/maximum values and standard deviation).

In order to determine the differences between the two groups regarding continuous variables, we used the nonparametric Mann-Whitney test, which was chosen because the study variables showed non-normal distribution. The OR was calculated with a confidence interval of $95 \%$. Adjustment for gender and age was made by ordinal logistic regression. For all analyses, we set the level of significance at 5\% and used the Statistical Package for Social Sciences, version 15.0 (SPSS Inc., Chicago, IL, USA).

\section{Results}

A total of 335 patients were invited to participate in the present study. Of those, 28 were excluded because they declined to undergo or failed to return for the reading of the results of any of the TSTs and 21 were excluded because they reported a history of tuberculosis. Therefore, the study sample consisted of 307 patients. The mean age was 53 years (range, 19-85 years), with a predominance of males (56.4\%), non-White individuals (65.4\%), married individuals (59.4\%), alcoholics (6.6\%), and smokers (88\%). Only 46 patients $(13.8 \%)$ were underweight (body mass index $\left.<18.5 \mathrm{~kg} / \mathrm{m}^{2}\right)$. Regarding comorbidities, 86 patients (28\%) had DM, 12 (4\%) were HCV-positive (although none were HBV-positive), and 2 (0.6\%) were HIV-positive. Only 9 patients (3\%) were using immunosuppressants. A total of 225 patients (73.4\%) had a BCG vaccination scar, and 63 $(20.6 \%)$ reported having had contact with a tuberculosis patients. Of the sample as a whole, $57.3 \%$ had no radiographic changes, whereas 42.7\% (131 patients) did. Of those, none were diagnosed with active tuberculosis.

The main causes of CKD were hypertensive nephrosclerosis (in 22.1\%), chronic glomerulonephritis (in 18.8\%), diabetic nephropathy (in 17.9\%), and other diseases (in 41.2\%). The etiology of CKD had little or no impact on the results of the TSTs, the chi-square test having revealed no significant differences among the results of TST1, TST2, and TST3 ( $p>0.05$ ).

When we adopted a cut-off induration $\geq 5 \mathrm{~mm}$ (for kidney transplant candidates, HIV-positive patients, immunosuppressed patients, and candidates for the use of TNF- $\alpha$ inhibitors), the prevalence of LTBI was $22.2 \%$ on TST1, increasing by $11.2 \%$ on TST2 (booster effect) and $2.8 \%$ on TST3 (TST conversion).

When we adopted a cut-off induration $\geq$ $10 \mathrm{~mm}$ (for CKD patients who were not kidney transplant candidates), the prevalence of LTBI was $28.5 \%$ on TST 1 , increasing by $9.4 \%$ on TST2 (booster effect) and 2.5\% on TST3 (TST conversion).

For the two cut-off indurations (of $5 \mathrm{~mm}$ and $10 \mathrm{~mm}$ ), the prevalence of LTBl increased significantly from TST1 to TST2, as well as from TST2 to TST3 ( $p<0.01$ for both). The cumulative frequency (TST1 and TST2) was 33.4\% (cut-off induration, $5 \mathrm{~mm}$ ) and 37.9\% (cut-off induration, $10 \mathrm{~mm}$ ), respectively (Table 1$)$. 
In our sample of patients with CKD, the MARI was $1.19 \%$.

There were no significant associations of age, etiology of CKD, hemodialysis efficiency, iron stores, use of vitamin D analogs, anemia, DM, malnutrition, date of hemodialysis initiation, duration of hemodialysis, HCV serology results, or presence of a BCG vaccination scar with the results of any of the TSTs ( $p>0.05$ ).

Risk factors such as smoking, alcoholism, DM, use of immunosuppressants, hypoalbuminemia, HIV infection, and absence of a BCG scar were evaluated in the study population, and we found no statistically significant associations between the tests and their respective cut-off points (TST1 induration $\geq 5 \mathrm{~mm}$ or $\geq 10 \mathrm{~mm}$, booster effect, and TST conversion) when evaluated in isolation. The TST result was more likely to be positive with a cut-off point of $10 \mathrm{~mm}$ in association with alcoholism and the male gender.

We found that TST1 induration was more likely to be $\geq 10 \mathrm{~mm}$ in males $(28 \%)$ than in females (15\%).

We found no statistically significant associations between the TST response and any of the following: maximum ferritin levels, as measured annually; mean ferritin levels, as calculated on the basis of four quarterly measurements (typically performed at the center for renal replacement therapy); transferrin saturation; albumin levels; and hemoglobin levels.

In comparison with nonsmokers, former smokers (31.1\%) showed a trend toward TST1 positivity (cut-off indurations of $5 \mathrm{~mm}$ and $10 \mathrm{~mm}$ ).

Having DM was not a significant risk factor for LTBI.

All of the CKD patients who were not vitamin D users showed a trend toward TST3 positivity $(p=0.033)$.

The LTBI treatment abandonment rate was $44.4 \%$. There were no adverse reactions that constituted an obstacle to the use of isoniazid.

\section{Discussion}

Between 2005 and 2006, 6 cases of tuberculosis occurred at our hemodialysis center, suggesting patient-to-patient transmission occurring in the cafeterias, in the procedure rooms, in the waiting rooms, or in the reception area. Therefore, it was important to determine the prevalence of $\mathrm{LTB} 1$ and the risk of infection in patients with CKD treated at our center.

Our sample of patients with CKD consisted mostly of non-White males, as has been reported elsewhere. ${ }^{(16)}$

We found that a positive TST result (i.e., a TST1 induration $\geq 10 \mathrm{~mm}$ ) was more likely to occur in males $(28 \%)$, a finding that is consistent with those of other studies..$^{(5,6)}$ We found no association between race and the TST response.

Nutritional data (including body mass index) and the etiology of CKD had no significant influence on the TST response, a finding that is consistent with those reported in previous studies. ${ }^{(5,6,8)}$

In the present study, having a history of contact with tuberculosis (20.6\%) did not coincide with the prevalence of tuberculosis infection, which was 33.4\% for a cut-off induration of 5 $\mathrm{mm}$ and $37.9 \%$ for a cut-off induration of 10 $\mathrm{mm}$ (cumulative frequency of TST1 and TST2). This can be explained by a lack of awareness of contact.

Our findings are consistent with those of a prevalence study involving elderly patients on hemodialysis. ${ }^{(5)}$ In that study, some of the patients had negative TST results (anergy), which were attributed to advanced age or immunological changes caused by uremia. ${ }^{(5)}$ Some of the negative TST results in our study might be related to uremia, given that the mean age of the participants was 53 years. Despite the limitations of the TST, the high prevalence of tuberculosis infection warrants the recommendation that the test be performed

Table 1 - Prevalence of latent tuberculosis infection in 307 patients with chronic kidney disease, Mineiro Institute of Nephrology, 2008-2009. ${ }^{\text {a }}$

\begin{tabular}{lcr}
\hline \multirow{2}{*}{ Results } & \multicolumn{2}{c}{ Patients in relation to the selected cut-off point for TST induration } \\
\cline { 2 - 3 } & $5 \mathrm{~mm}$ & $10 \mathrm{~mm}$ \\
\hline Negative & $46(63.8)$ & $140(59.6)$ \\
Positive & $16(22.2)$ & $67(28.5)$ \\
Booster effect & $8(11.2)^{*}$ & $22(9.4)^{*}$ \\
TST conversion & $2(2.8)^{*}$ & $6(2.5)^{*}$ \\
TOTAL & $72(100.0)$ & $235(100.0)$ \\
\hline
\end{tabular}

TST: tuberculin skin test. ${ }^{a}$ Values expressed as $n(\%) .{ }^{*}$ Chi-square test $(p<0.05)$. 
more carefully in CKD patients, who are at a high risk of active tuberculosis. ${ }^{(5,8)}$

The $12.7 \%$ increase in the prevalence of LTB1 from TST1 to TST2 and the 5\% increase in the prevalence of LTB1 from TST2 to TST3 were statistically significant $(p<0.01)$, suggesting the importance of a second test for early detection of LTBI and a third test for those who show no booster effect. The TST conversion was probably due to contact in the community, given that the incidence of tuberculosis in the city of Belo Horizonte is high ( $>30 / 100,000$ population), according to the World Health Organization, ${ }^{(16)}$ as well as to the fact that there were no cases of active (transmissible) tuberculosis at our center during the study period.

Some of the relevant findings of the present study include the fact that the prevalence of LTBI in patients with CKD was high and the fact that TST2 and TST3 significantly increased the detection of LTBI in individuals who had initially tested negative.

Various studies have found that patients with CKD are at a high risk of developing tuberculosis, a finding that is inconsistent with those of the present study and that is probably due to the use of isoniazid in those patients who completed the preventive treatment or to the short follow-up period..$^{(10,11,17,18)}$ The LTBI treatment abandonment rate found in the present study (i.e., 44.4\%) is consistent with the rate reported by other authors, ${ }^{(19)}$ reinforcing the need for health policies aimed at improving treatment adherence in such patients.

In the present study, we found no association between cases of active tuberculosis and any of the following: hemoglobin and hematocrit levels; iron stores; and positive TST results (LTBI). Genetic disorders in humans, such as mutations in ferroportin 1, can increase the risk of tuberculosis or negatively interfere with the response to treatment if there is an increase in the supply of iron to the macrophage. Therefore, iron status can play an important role in tuberculosis prevention and treatment, as well as in the development of tuberculosis vaccines. ${ }^{(20)}$

In the present study, we found no significant association of the study variables (age, being married, etiology of CKD, dialysis efficiency, iron stores, anemia, DM, malnutrition, date of hemodialysis initiation, HCV serology results, and presence of a BCG vaccination scar) with the results of the TSTs (TST1, TST2, and TST3) when analyzed in isolation, a finding that is consistent with those of other studies. ${ }^{(5,18)}$

False-positive reactions can occur in individuals vaccinated with BCG, especially after the first year of vaccination, when the vaccine produces reactions that are stronger and last longer. However, the reaction tends to decrease over time, and, if the TST is performed within 10 years or more after the last vaccination, the effect of BCG vaccination might be minimal. This probably did not affect the results of the present study, given that, in Brazil, individuals usually receive BCG vaccination in the first days of life. ${ }^{(2)}$

Vitamin D deficiency probably had no role in reducing the cellular response (negative TST), ${ }^{(19)}$ given that patients with vitamin D deficiency tended to show positive TST3 results $(p=0.033)$.

Although studies have shown an association of tuberculosis with alcoholism and smoking, ${ }^{(21,22)}$ our study showed no association of those variables with tuberculosis or LTBl, the exception being an association of tuberculosis and LTBI with the male gender and alcohol consumption when a cut-off induration of $10 \mathrm{~mm}$ was adopted.

In the CKD patients investigated in the present study, the MARI was similar to that reported for the general population of Brazil, which suggests recent infection. The highest MARI in the world is found in sub-Saharan Africa (1.5-2.5\%). In developed countries, the MARI is $0.1 \%$. In South America, the MARI has been estimated at 0.5-1.5\%. In Brazil, the MARI is currently 1\%, being lower in the southern region of the country and larger in the southeastern, northern, and northeastern regions. ${ }^{(19)}$

Although gamma interferon release assays and TSTs are similar in terms of their ability to detect LTBI, the relatively high cost and the need for laboratory infrastructure are some of the limitations of the former. ${ }^{(23)}$ There is a lack of data on the use of gamma interferon release assays for detecting LTBI in patients with CKD undergoing hemodialysis. ${ }^{(9,24)}$ In Brazil, interferon gamma release assays have yet to be more widely employed as a method for diagnosing LTB1. ${ }^{(3,12)}$

In the present study, the TST proved to be a useful tool for the detection of LTBI in patients with CKD undergoing hemodialysis. We can conclude that the prevalence of LTBI in the study population was high and the MARI was similar to that reported for the general population of Brazil, which suggests recent infection. 


\section{References}

1. WHO global tuberculosis control report 2010. Summary. Cent Eur J Public Health. 2010;18(4):237.

2. Secretaria de Vigilância em Saúde. Informe eletrônico da tuberculose. Boletim Eletrônico Epidemiológico. 2009;9(2):1-4.

3. Conde MB, Melo FA, Marques AM, Cardoso NC, Pinheiro VG, Dalcin Pde T, et al. Ill Brazilian Thoracic Association Guidelines on tuberculosis. J Bras Pneumol. 2009;35(10):1018-48.

4. Girndt M, Sester M, Sester U, Kaul H, Köhler H. Defective expression of B7-2 (CD86) on monocytes of dialysis patients correlates to the uremia-associated immune defect. Kidney Int. 2001;59(4):1382-9.

5. Wauters A, Peetermans WE, Van den Brande P, De Moor B, Evenepoel P, Keuleers H, et al. The value of tuberculin skin testing in haemodialysis patients. Nephrol Dial Transplant. 2004;19(2):433-8.

6. Woeltje KF, Mathew A, Rothstein M, Seiler S, Fraser VJ. Tuberculosis infection and anergy in hemodialysis patients. Am J Kidney Dis. 1998;31(5):848-52.

7. Smirnoff M, Patt C, Seckler B, Adler JJ. Tuberculin and anergy skin testing of patients receiving long-term hemodialysis. Chest. 1998;113(1):25-7.

8. Shankar MS, Aravindan AN, Sohal PM, Kohli HS, Sud K, Gupta KL, et al. The prevalence of tuberculin sensitivity and anergy in chronic renal failure in an endemic area: tuberculin test and the risk of post-transplant tuberculosis. Nephrol Dial Transplant. 2005;20(12):2720-4.

9. Maden E, Bekci TT, Kesli R, Atalay H, Teke T, Solak Y, et al. Evaluation of performance of quantiferon assay and tuberculin skin test in end stage renal disease patients receiving hemodialysis. New Microbiol. 2011;34(4):351-6.

10. Hussein MM, Mooij JM, Roujouleh H. Tuberculosis and chronic renal disease. Semin Dial. 2003;16(1):38-44.

11. Lundin AP, Adler AJ, Berlyne GM, Friedman EA. Tuberculosis in patients undergoing maintenance hemodialysis. Am J Med. 1979;67(4):597-602.

12. Ministério da Saúde. Secretaria de Vigilância em Saúde. Departamento de Vigilância Epidemiológica. Manual de recomendações para o controle da tuberculose no Brasil. Brasília: Ministério da Saúde; 2011.

13. Targeted tuberculin testing and treatment of latent tuberculosis infection. This official statement of the American Thoracic Society was adopted by the ATS Board of Directors, July 1999. This is a Joint Statement of the American Thoracic Society (ATS) and the Centers for Disease Control and Prevention (CDC). This statement was endorsed by the Council of the Infectious Diseases Society of America. (IDSA), September 1999, and the sections of this statement. Am J Respir Crit Care Med. 2000;161(4 Pt 2):S221-47.

14. Manuel O, Humar A, Preiksaitis J, Doucette K, Shokoples $\mathrm{S}$, Peleg AY, et al. Comparison of quantiferon-TB gold with tuberculin skin test for detecting latent tuberculosis infection prior to liver transplantation. Am J Transplant. 2007;7(12):2797-801.

15. Ruffino-Netto A. Epidemiometric models in tuberculosis. Definition of "states" and risk of infection [Article in Portuguese]. Rev Saude Publica. 1977;11(2):188-98.

16. Guilbert JJ. The world health report 2002 - reducing risks, promoting healthy life. Educ Health (Abingdon). 2003;16(2):230.
17. Mazurek GH, Jereb J, Vernon A, LoBue P, Goldberg S, Castro K, et al. Updated guidelines for using Interferon Gamma Release Assays to detect Mycobacterium tuberculosis infection - United States, 2010. MMWR Recomm Rep. 2010;59(RR-5):1-25.

18. Klote MM, Agodoa LY, Abbott KC. Risk factors for Mycobacterium tuberculosis in US chronic dialysis patients. Nephrol Dial Transplant. 2006;21(11):3287-92.

19. Kritski AL, Conde MB, Souza MG, editors. Tuberculose: do ambulatório à enfermaria. 3rd ed. São Paulo: Atheneu; 2005.

20. Boelaert JR, Vandecasteele SJ, Appelberg R, Gordeuk VR. The effect of the host's iron status on tuberculosis. J Infect Dis. 2007;195(12):1745-53.

21. Caron-Ruffino M, Ruffino-Netto A. Association of alcoholism and pulmonary tuberculosis [Article in Portuguese]. Rev Saude Publica. 1979;13(3):183-94.

22. Sopori ML, Kozak W. Immunomodulatory effects of cigarette smoke. J Neuroimmunol. 1998;83(1-2):148-56.

23. Dagnew AF, Hussein J, Abebe M, Zewdie M, Mihret A, Bedru A, et al. Diagnosis of latent tuberculosis infection in healthy young adults in a country with high tuberculosis burden and BCG vaccination at birth. BMC Res Notes. 2012;5:415.

24. Herrera V, Perry S, Parsonnet J, Banaei N. Clinical application and limitations of interferon-gamma release assays for the diagnosis of latent tuberculosis infection. Clin Infect Dis. 2011;52(8):1031-7. 


\section{About the authors}

\section{Jane Corrêa Fonseca}

Nephrologist. Mineiro Institute of Nephrology, Belo Horizonte, Brazil.

\section{Waleska Teixeira Caiaffa}

Full Professor. Department of Public Health, Federal University of Minas Gerais School of Medicine, Belo Horizonte, Brazil.

\section{Mery Natali Silva Abreu}

Adjunct Professor. Department of Statistics. Federal University of Minas Gerais School of Nursing, Belo Horizonte, Brazil.

\section{Katia de Paula Farah}

Adjunct Professor. Federal University of Minas Gerais School of Medicine, Belo Horizonte, Brazil.

\section{Wânia da Silva Carvalho,}

Associate Professor 1. Department of Social Pharmacy, Federal University of Minas Gerais School of Pharmacy, Belo Horizonte, Brazil.

\section{Silvana Spindola de Miranda}

Associate Professor IV. Federal University of Minas Gerais School of Medicine, Belo Horizonte, Brazil. 